\section{Klinische Bedeutung von Arzneimittelinteraktionen Überlegt therapieren - Risiko minimieren}

U nerwünschte Arzneimittelwirkungen (UAWs) sind für 2-6\% aller stationären Krankenhausaufnahmen verantwortlich $(1,2)$ - wie verschiedene Studien zeigen. Besonders gefährdet sind ältere Patienten. Untersuchungen aus Jena und Rostock kommen zu dem Ergebnis, dass in der Gruppe der über 70-Jährigen zwei von 1000 ambulanten Patienten wegen einer UAW zur stationären Aufnahme kommen. Nach dieser Untersuchung werden UAWs am häufigsten durch kardiovaskuläre Pharmaka (Digitalis, Kalziumkanalblocker, ACEHemmer etc.) verursacht; es folgen Antithrombotika, NSAR und Antidiabetika. Die häufigsten Symptome der UAW sind bei den kardiovaskulären Pharmaka Bradykardie, Synkopen und Arrhythmien. In der Gruppe der NSAR und Antithrombotika werden am häufigsten gastrointestinale Blutungen beobachtet. Ursachen für UAWs sind

- das Nichtbeachten von Kontraindikationen

- falsche Dosierung und

- Arzneimittelinteraktionen.

Bis zu 22\% aller UAWs sind Arzneimittelinteraktionen zuzuordnen. Nach einer französischen Untersuchung werden sehr häufig Arzneistoffe miteinander kombiniert, die jeweils eine Verlängerung der QT-Zeit bewirken (3). Bei Patienten, die in die Notfallaufnahme kommen, besteht im Mittel bereits eine Medikation mit 7,2 Arzneistoffen, die in 25\% der Fälle potenzielle Interaktionen beinhalten (4). Auch bei Entlassung aus dem Krankenhaus erhalten Patienten häufig (60\%) Arzneimittelkombinationen, die Interaktionen beinhalten; in $12 \%$ der Fälle sind diese als schwerwiegend einzuordnen (5). Unberücksichtigt bleibt die Selbstmedikation. Seit den Erkenntnissen mit Johanniskraut sollte die Einnahme von Phytopharmaka in der Arzneimittelanamnese abgefragt werden. Johanniskraut ist ein potenter Induktor des CytochromP450-Isoenzyms CYP3A4 und vermindert unter anderem den Serum- spiegel von Ciclosporin, Nevirapin, Indinavir und Simvastatin (6).

Unkritisches Verordnen mehrerer Medikamente vermeiden

UAWs können einen Krankenhausaufenthalt herbeiführen, diesen verlängern und die Behandlungskosten erhöhen. Auf Basis der eingangs erwähnten Zahlen aus Jena und Rostock (2,4\% der Krankenhausaufnahmen durch UAWs) verursachen die UAW-bedingten Krankenhauseinweisungen in der BRD direkte Mehrkosten von zirka 400 Millionen Euro; falls 20\% davon auf Arzneimittelinteraktionen zurückzuführen sind, ergibt dies einen Mehraufwand von mindestens 80 Millionen Euro.

UAWs durch Arzneimittelinteraktionen können minimiert werden. Voraussetzungen sind Kenntnisse über die pharmakologischen Wirkungen (zur Vermeidung pharmakodynamischer Interaktionen), der Pharmakokinetik (Halbwertszeit, Dosisanpassung bei eingeschränkter Nieren- und Leberfunktion) und der Metabolisierungswege (zur Vermeidung pharmakokinetischer Interaktionen) der verordneten Arzneistoffe. Da niemand diese pharmakologischen Daten aller Arzneimittel sicher kennen kann, ist es sinnvoll, sich in der medikamentösen Therapie auf eine begrenzte Anzahl von Medikamenten zu beschränken. Ebenfalls sollte das unkritische Verordnen einer Vielzahl von Medikamenten vermieden werden. Die Inzidenz von UAWs und Arzneimittelinteraktionen nimmt exponentiell mit der Zahl der verordneten Pharmaka zu. In den verschiedenen Arzneimittelgruppen (z.B. CSE-Hemmstoffe, Antibiotika, Protonenpumpenhemmer) gibt es meistens eine oder mehrere Substanzen mit geringerem Interaktionspotenzial. Diese sind zu bevorzugen. Bei multimorbiden Patienten, die zahlreiche Medikamente erhalten müssen, sind UAWs und Arzneimittelinteraktionen nicht immer vermeidbar. Bei diesen Patienten ist es notwendig, möglichst früh an eine Arzneimittelinteraktion zu denken, um schwerwiegende UAWs zu vermeiden.

\section{Fazit für die Praxis}

Bis zu 25\% aller UAWs sind durch Arzneimittelinteraktionen bedingt. UAWs durch Arzneimittelinteraktionen können bei Kenntnis der pharmakologischen Wirkungen, der Pharmakokinetik (Dosisanpassung bei eingeschränkter Nieren- und Leberfunktion) und der Metabolisierungswege der Arzneistoffe minimiert werden. Zur Vermeidung von Interaktionen sollte die möglichst minimale Anzahl an Wirkstoffen verordnet werden. In einer Pharmakagruppe sollte eine Substanz mit möglichst geringem Interaktionspotenzial ausgesucht werden.

\section{Literatur}

1. Schneeweiss S, Hasford J, Göttler M et al. Admissions caused by adverse drug events to internal medicine and emergency departments in hospitals: a longitudinal population-based study. Eur J Clin Pharmacol 2002; 58: 285-291

2. Lazarou J, Pomeranz BH, Corcy PN. Incidence of adverse drug reactions in hospilized patients. JAMA 1998; 279: 1200-1205

3. Guedon-Moreau L, Ducrocq D, Duc MF et al. Absolute contraindications in relation to potential drug interactions in outpatient prescriptions: analysis of the first five million prescriptions in 1999. Eur J Clin Pharmacol 2003; 59: 689-695

4. Gaddis GM, Holt TR, Woods M. Drug interactions in at-risk emergency department patients. Acad Emerg Med 2002; 9 (11): 1162-1167

5. Egger SS, Drewe J, Schlienger RG. Potential drug-drug interactions in the medication of medical patients at hospital discharge. Eur J Clin Pharmacol 2003; 58: 773 778

6. Klotz U, Beil W, Gleiter C et al. Arzneimittelinteraktionen Mechanismen und klinische Bedeutung. Der Internist 2003; 44: 1444-1449

\section{Anschrift des Verfassers}

Prof. Dr. med. W. Beil Institut für Pharmakologie

Medizinische Hochschule Hannover Carl-Neuberg-Str. 1

30625 Hannover

In der nächsten Ausgabe der Notfall \& Hausarztmedizin lesen Sie von Prof. Beil „Mechanismen von Arzneimittelinteraktionen“. 